\title{
Direct and Indirect Costs of Influenza-Like Illness Treated with and Without Oseltamivir in 15 European Countries: A Descriptive Analysis Alongside the Randomised Controlled ALIC ${ }^{4}$ Trial
}

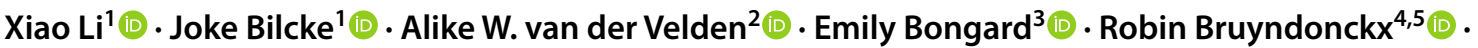

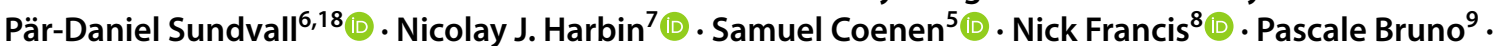 \\ Ana Garcia-Sangenis ${ }^{10}$ (1) Dominik Glinz ${ }^{11}$ - Katarzyna Kosiek $^{12} \cdot$ Réka Mikó-Pauer $^{13}$. \\ Ruta Radzeviciene Jurgute ${ }^{14} \cdot$ Bohumil Seifert $^{15}$ (D) . Nikolaos Tsakountakis ${ }^{16} \cdot$ Rune Aabenhus $^{17}$ (1) . \\ Christopher C. Butler ${ }^{3} \cdot$ Philippe Beutels ${ }^{1}$ (I)
}

Accepted: 20 June 2021 / Published online: 22 July 2021

(c) The Author(s) 2021

\begin{abstract}
Background and Objective Influenza-like illness (ILI) leads to a substantial disease burden every winter in Europe; however, oseltamivir is not frequently prescribed to ILI patients in the primary-care setting. An open-label, multi-country, multi-season, randomised controlled trial investigated the effectiveness of oseltamivir for treating ILI in 15 European countries. We aimed to evaluate whether patients presenting with ILI in primary care and being managed with the addition of oseltamivir to usual care had lower average direct and indirect costs compared to patients with usual care alone.

Methods Resource use data were extracted from participants' daily diaries. Itemised country-specific unit costs were collected through official tariffs, pharmacies or literature. Costs were converted to 2018 values. The null hypothesis was tested based on one-sided credible intervals (CrIs) obtained by bootstrapping. Base-case analysis estimated direct cost and productivity losses using itemised costed resource use and the human capital approach. Scenario analyses with self-reported spending rather than itemised costing were also performed.

Results Patients receiving oseltamivir $(N=1306)$ reported fewer healthcare visits, medication uses, hospital attendances and paid-work hours lost than the other patients $(N=1298)$. Excluding the oseltamivir cost, the average direct costs were lower in patients treated with oseltamivir from all perspectives, but these differences were not statistically significant (perspective of patient: $€ 17$ [0-95\% Crl: 16-19] vs. $€ 24$ [5-100\% Crl: 18-29]; healthcare provider: $€ 37$ [28-67] vs. $€ 44$ [25-55]; healthcare payers: €54 [45-85] vs. €68 [45-81]; and society: €423 [399-478] vs. €451 [390-478]). Scenario and age-group analyses confirmed these findings, but with some between-country differences.

Conclusion The average direct and indirect costs were consistently lower in patients treated with oseltamivir than in patients without from four perspectives (excluding the oseltamivir cost). However, these differences were not statistically significant.
\end{abstract}

\section{Introduction}

There were an estimated 145,000 deaths and 9.5 million hospitalisations worldwide from influenza virus infections in 2017 [1]. Many other respiratory viruses cause clinical symptoms that are often indistinguishable on clinical grounds alone. In primary care, patients with influenza-like symptoms are often managed as influenza-like illness (ILI)

Xiao Li

xiao.li@uantwerpen.be

Extended author information available on the last page of the article without any diagnostic testing, where ILI is defined by the World Health Organization (WHO) as an acute respiratory infection with measured fever of $\geq 38^{\circ} \mathrm{C}$ and cough, with onset within the last 10 days [2]. Furthermore, ILI peaks in the northern hemisphere winter together with many other viral illnesses, adding to pressure on the healthcare system and society [3].

The direct treatment costs of ILI are borne by both patients and healthcare providers (e.g. national health services or public health insurance funds). Moreover, indirect costs arise for society when patients are unable to be productive at work, or perform their usual activities [4]. 


\section{Key Points}

Patients receiving oseltamivir reported fewer healthcare resource use items (including healthcare visits, medications, hospital attendances) and paid-work hours lost than patients receiving usual care alone.

Excluding the costs of oseltamivir, the average direct costs were lower in patients treated with oseltamivir from the patients', healthcare providers', healthcare payers' and societal perspectives, but these differences were not statistically significant.

Our findings provide valuable information to decisionmakers and researchers on ILI-associated costs and treating patients with oseltamivir on a European and country-level from four different perspectives, especially for budgetary considerations and subsequent cost-effectiveness analysis.

In 2002, a neuraminidase inhibitor, oseltamivir (brand name Tamiflu ${ }^{\circledR}$ ), was licensed for treating influenza in the European Union. However, oseltamivir is not frequently prescribed in primary care in Europe, partly because clinical guidelines only recommend oseltamivir for 'at-risk' groups (e.g. patients with underlying conditions) to be started within $48 \mathrm{~h}$ of symptom onset, or based on the treating physician's risk-assessment [5-8].

Several meta-analyses of randomised trial findings demonstrated that, compared with placebo, oseltamivir reduces the time to first alleviation of symptoms [9-12]. Nevertheless, the applicability of these findings to everyday world clinical practice is uncertain, because most of the clinical trials were industry sponsored and children, older people, and those with co-morbidities were under-represented. Furthermore, the effects of oseltamivir on resource use, productivity losses and health-related quality of life (HRQoL) are still largely unknown.

An investigator-initiated, open-label, pragmatic trial evaluated the effect of oseltamivir on ILI in a real-world setting $\left(\mathrm{ALIC}^{4} \mathrm{E}\right.$ : Antivirals for influenza-Like Illness? A randomised Controlled trial of Clinical and Cost effectiveness in primary CarE, registry number: ISRCTN 27908921). $\mathrm{ALIC}^{4} \mathrm{E}$ found that the time to recovery was significantly shorter in patients treated with oseltamivir, with an overall absolute average benefit of 1.02 days (95\% Bayesian credible interval 0.74-1.31) [13].

As a secondary analysis of the $\mathrm{ALIC}^{4} \mathrm{E}$, using resource use information collected in the trial, as well as unit costs sourced external to the trial, the primary objectives of this study were (1) to estimate the average cost per ILI patient treated with and without oseltamivir, and (2) to determine whether ILI patients treated with oseltamivir incur lower average costs than ILI patients treated without, when the cost of oseltamivir was not included. We also explored the reasons for potential differences in healthcare resource use and loss of activities between ILI patients treated with and without oseltamivir as a secondary objective. Four perspectives were considered in this analysis: patient, healthcare provider, healthcare payers (patient + healthcare provider) and society.

\section{Methods}

\subsection{Brief Description of the Trial}

$\mathrm{ALIC}^{4} \mathrm{E}$ was an open-label, randomised, controlled trial conducted in 15 European countries (21 networks covering 209 primary care practices) over three consecutive winters (Q4 2015-Q2 2018) [13, 14]. Patients were recruited via primary care practices when they presented with ILI symptoms (duration $\leq 72 \mathrm{~h}$ ) during seasonal influenza epidemics. The eligible patients were randomly assigned at the point of care into two groups in a 1 to 1 ratio: usual primary care or usual primary care with the addition of oseltamivir, stratified by age, severity of symptoms, co-morbidity and duration of symptoms onset. Oseltamivir was given twice a day for 5 days. The dosage was $75 \mathrm{mg}$ for patients aged $\geq 13$ years, or according to their weights for patients $\geq 1$ year and $<13$ years of age $[14,15]$.

\subsection{Resource-Use Data}

Every day, patients reported on the use of 11 types of prescribed and over-the-counter (OTC) medication (Table 1 (b), medication use) in a diary (completed by a caregiver for patients aged $\leq 12$ years) over 2 weeks. On days 7 and 14, patients also reported on the number of healthcare visits, amount of money they spent in association with their ILI, number of hours missed from their usual activities and work, number of hospital attendances, and length of stay (LoS) if admitted (Table 1). Participants were telephoned on days 14 and 28 and asked questions on clinical symptoms, hospital attendances and LoS, and were encouraged to complete and return their diaries. We report the hospital attendances from days 14 to 28 , but excluded them from the cost analysis, because no data were available on healthcare visits and medication use for that period. Study diaries and telephone questionnaires can be found in the Supplemental Material, Online Resources 2, 3 and 4.

\subsection{Unit Costs for Resource-Use Data}

Unit costs were collected in three steps. Firstly, a unit cost template was designed (for adults and children separately 
and corresponding to the resource use categories) and sent out to all national network teams to collect country-specific data. A phone interview was conducted with a network team member from each participating country for information about the country's healthcare system and reimbursement of healthcare expenditure. Secondly, country-specific costs of primary and secondary healthcare visits, medication and hospital admissions were collected via national tariffs, governmental reports and websites, online pharmacies and literature. Conservative assumptions were made if data were unavailable (e.g. when the unit cost of out-of-hour service was unavailable, the unit cost of a general practitioner (GP) visit was assumed). Lastly, after a country's unit cost collection was completed, the unit cost dataset was shared with the country's team representative and with health economists in the UK, Belgium and Sweden to validate the data and assumptions in an iterative process ( $1-4$ iterations).

The average price of each type of healthcare visit and medication item was collected, and if unavailable, we used the most frequently cited price. Costs retrieved from the literature were inflated and converted to Euro year 2018 values using the World Bank's country-specific consumer price indices (CPI), and Eurostat's purchasing power parities (PPPs) [16, 17]. The human capital approach was used to calculate productivity losses by paid-work hours lost (see Supplemental Material, Online Resource 1, section 1).

\subsection{Base-Case Analysis}

For the base-case analysis, the average cost for an ILI patient was considered from four perspectives using tariffs and prices for unit costs over a time horizon of 14 days. The societal perspective in the base case included productivity losses of paid work. The cost of oseltamivir is not included in base-case or scenario analysis, unless explicitly stated otherwise. If a patient reported any use (including single time use) of any kind of medication, the base-case assumption is that they purchased a new package from a pharmacy, because cost per dose could not be estimated. We assumed that a patient not reporting a particular healthcare use or cost (not available/blank) did not incur this type of healthcare use or cost.

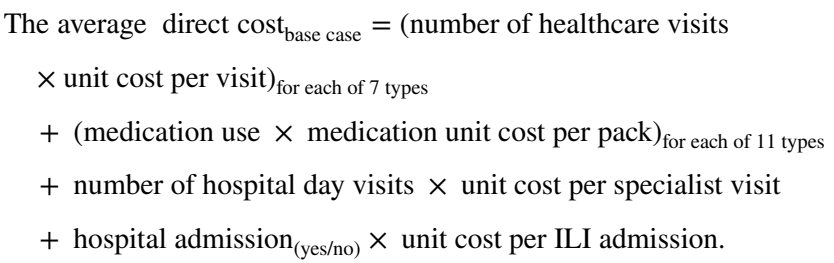

The average total cost $_{\text {base-case }}=$ the average direct cost $t_{\text {base-case }}$ + paid-work hour losses $\times$ national average wage per hour.
Table 1 listed the seven types of healthcare visits and 11 types of medication.

\subsection{Scenario Analysis}

In scenario analysis we used the patient's reported direct and indirect expenditures (Table 1(e)) to estimate average direct and total cost. As such, we only included indirect expenditures and personal income losses reported by patients, not the full opportunity cost of lost productive time to employers and society, hence the perspective here is considered to be a partial societal perspective", and not fully comparable to the societal perspective in the base-case analysis.

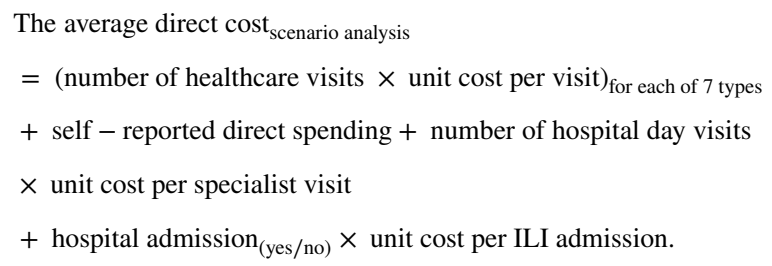

The average total cost $t_{\text {scenario analysis }}$

$=$ the average direct $\operatorname{cost}_{\text {scenario analysis }}$

+ indirect spending.

\subsection{Age Group and Country-Specific Analysis}

Average direct and total cost for different perspectives were also obtained for adults/adolescents (patients aged $\geq 13$ years) and children (aged $<13$ years) separately, as well as by country.

\subsection{Statistical Analysis}

All the data analyses were carried out using R version 3.5.0. We tested whether the average cost per ILI patient treated with oseltamivir is lower than for an ILI patient treated without oseltamivir, using the following one-sided hypothesis:

$\mathrm{H}_{0}$. average cost per ILI patient treated with oseltamivir is equal to or higher than average cost per ILI patient treated without oseltamivir.

$\mathrm{H}_{1}$ : average cost per ILI patient treated with oseltamivir is lower than average cost per ILI patient treated without oseltamivir.

A one-sided hypothesis test is appropriate, because an intervention associated with higher average direct and total costs, without including the cost of the intervention, is less likely to be recommended by decision makers. We used a nonparametric bootstrap method to test this hypothesis, to account for the positive and right-skewed distribution of the cost data. We bootstrapped 10,000 replications (using 
R package: 'boot', percentile method) to estimate one-sided 95\% credible intervals ( $\mathrm{CrIs}$ ) around the average direct and indirect costs $[18,19]$. The null hypothesis is rejected if the one-sided CrIs do not overlap (oseltamivir group: 5-100\% CrI vs. usual care group: $0-95 \% \mathrm{CrI}$ ). Note that using nonoverlapping CrIs is equivalent to using a one-sided $p$ value below 0.05 as a test for significance.

For simplicity, we refer in the text below to 'the usual care group' and the 'oseltamivir group', although both groups received usual care, but in the latter group it was supplemented with oseltamivir.

\section{Results}

About half of the 2595 trial participants (2212 (85\%) adults/ adolescents and 383 (15\%) children) were assigned to the oseltamivir group (Fig. 1 and Supplemental Material, Online Resource 1, Table 9).

\subsection{Average Resource Use per Influenza-Like Illness (ILI) Patient}

The descriptive analysis (without formal testing for significant differences) in Table 1 (a) shows that after the initial recruitment visit, the average number of subsequent healthcare visits per type were either similar or lower in the oseltamivir group compared with the usual care group, except for the telephone consultations (0.02 vs. 0.01 per ILI patient, respectively). GP visits were the most frequent type of healthcare visit in both groups (71\% and $74 \%$ ), and the average number of GP visits per patient were 0.85 and 0.89 , respectively. Accident and Emergency (A\&E) was the least frequent type of visit (only $1 \%$ in both groups).

Slightly fewer patients treated with oseltamivir reported any use of additional medication than patients treated with usual care only (95\% vs. $96 \%$ ). Each type of medication was also used by slightly fewer patients in the oseltamivir group compared to the usual care group, and $74 \%$ patients with oseltamivir reported paracetamol use versus $77 \%$ patients with usual care alone (Table 1(b)).

Fewer hospital attendances were recorded in the oseltamivir group, both for outpatient visits (47 vs. 56) and for admissions (8 vs. 14). LoS during the first 2 weeks after recruitment was shorter for patients from the oseltamivir group (4.5 vs. 5.8 days) (Table 1(c)). During the third and fourth weeks, two patients with oseltamivir reported longer LoS than one patient with usual care. One patient reported an admission (without a discharge date) due to a suspected unexpected serious adverse reaction (SUSAR, categorized as "might be possibly related to oseltamivir", a leg amputation below the knee). We excluded this patient when estimating number of admission and LoS during weeks 3 and 4, because this SUSAR was very rare and the trial was not powered to detect such a rare event.

Patients in both groups reported a similar percentage of any activity loss $(78 \%$, Table $1(\mathrm{~d}))$. The reported average hours of activity loss were lower in the oseltamivir group than in the usual-care group, except for voluntary work $(0.53$ vs. $0.49 \mathrm{~h})$. More than two-thirds of patients reported paid-work loss. On average, patients treated with oseltamivir reported 40 minutes less absenteeism from paid work than patients treated with usual care (20.25 vs. $20.93 \mathrm{~h}$ ).

Fewer patients treated with oseltamivir reported any spending for their ILI episode than patients treated with usual care (63\% vs. 68\%, Table 1(e)). Patients treated with oseltamivir reported lower average direct ( $€ 10.64$ vs. 14.57) and indirect expenditures (€5.57 vs. 8.25) for ILI than patients treated with usual care (Table 1(e)). Resource use per ILI patient by age group and by country are presented in the Supplemental Material, Online Resource 1.
Fig. 1 Flow chart of recruited patients

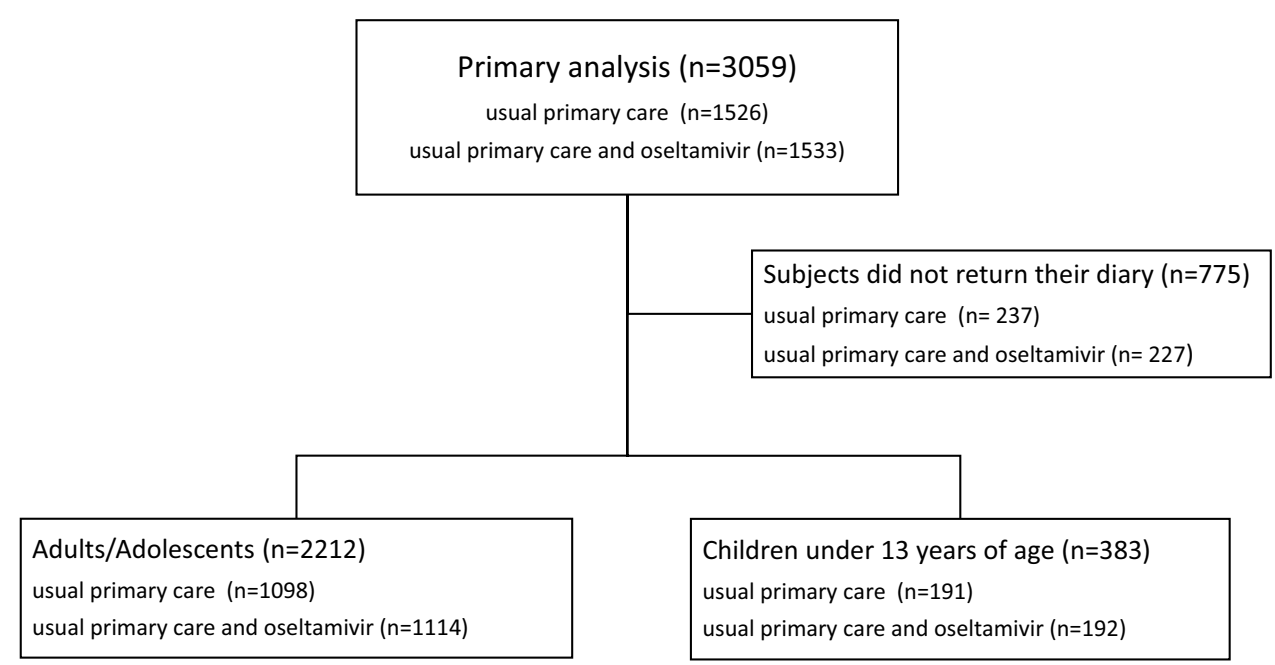




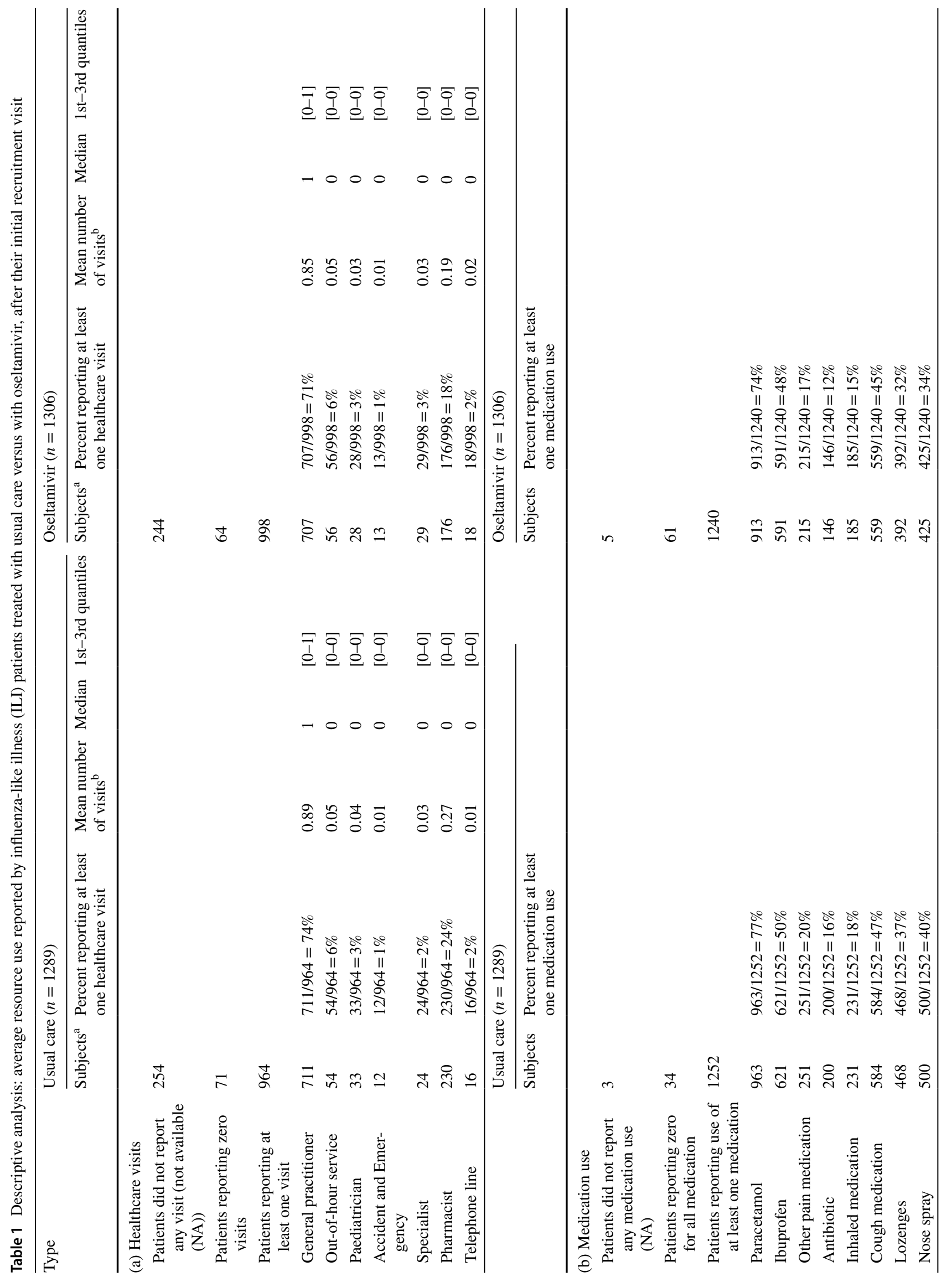




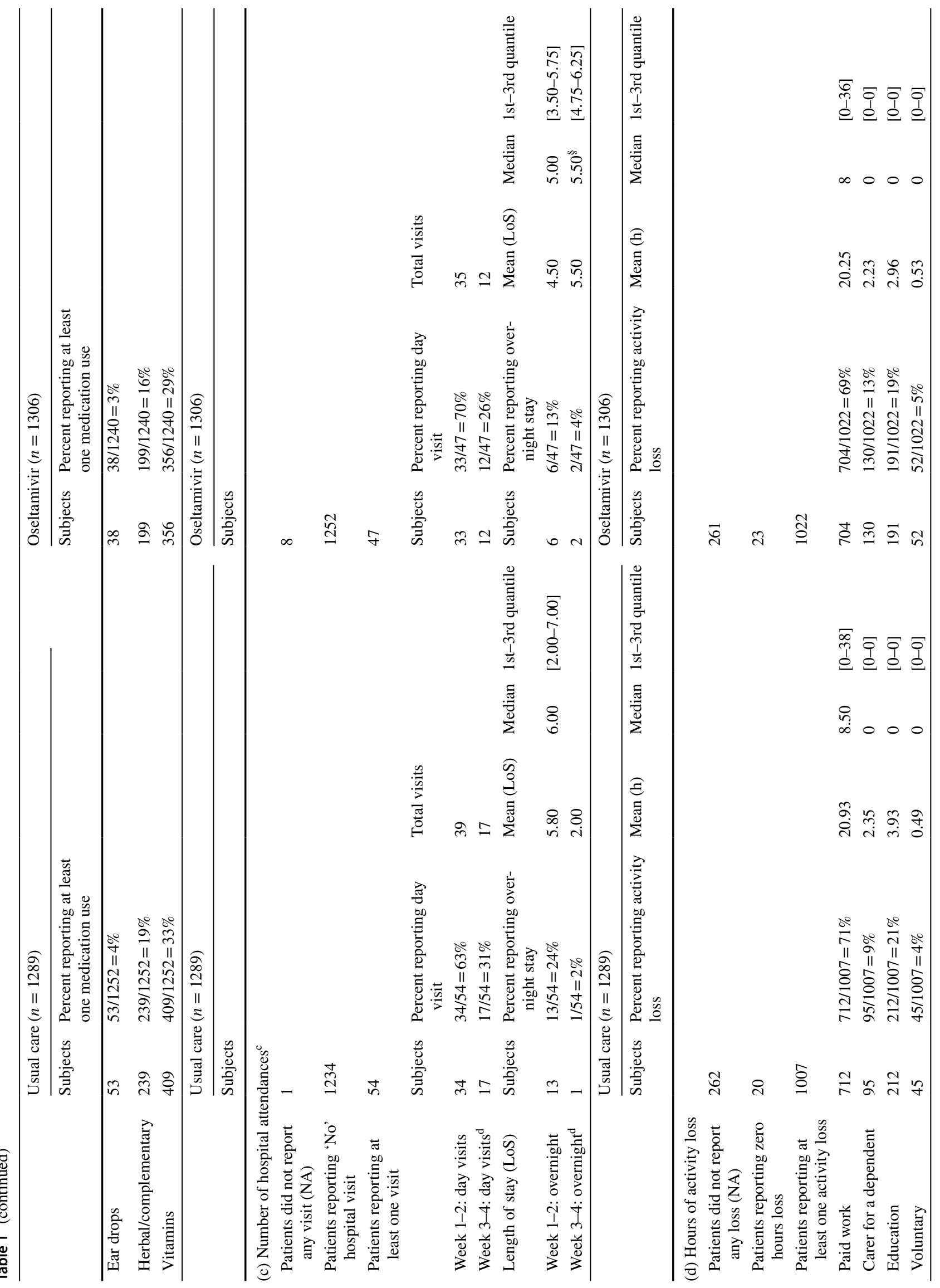




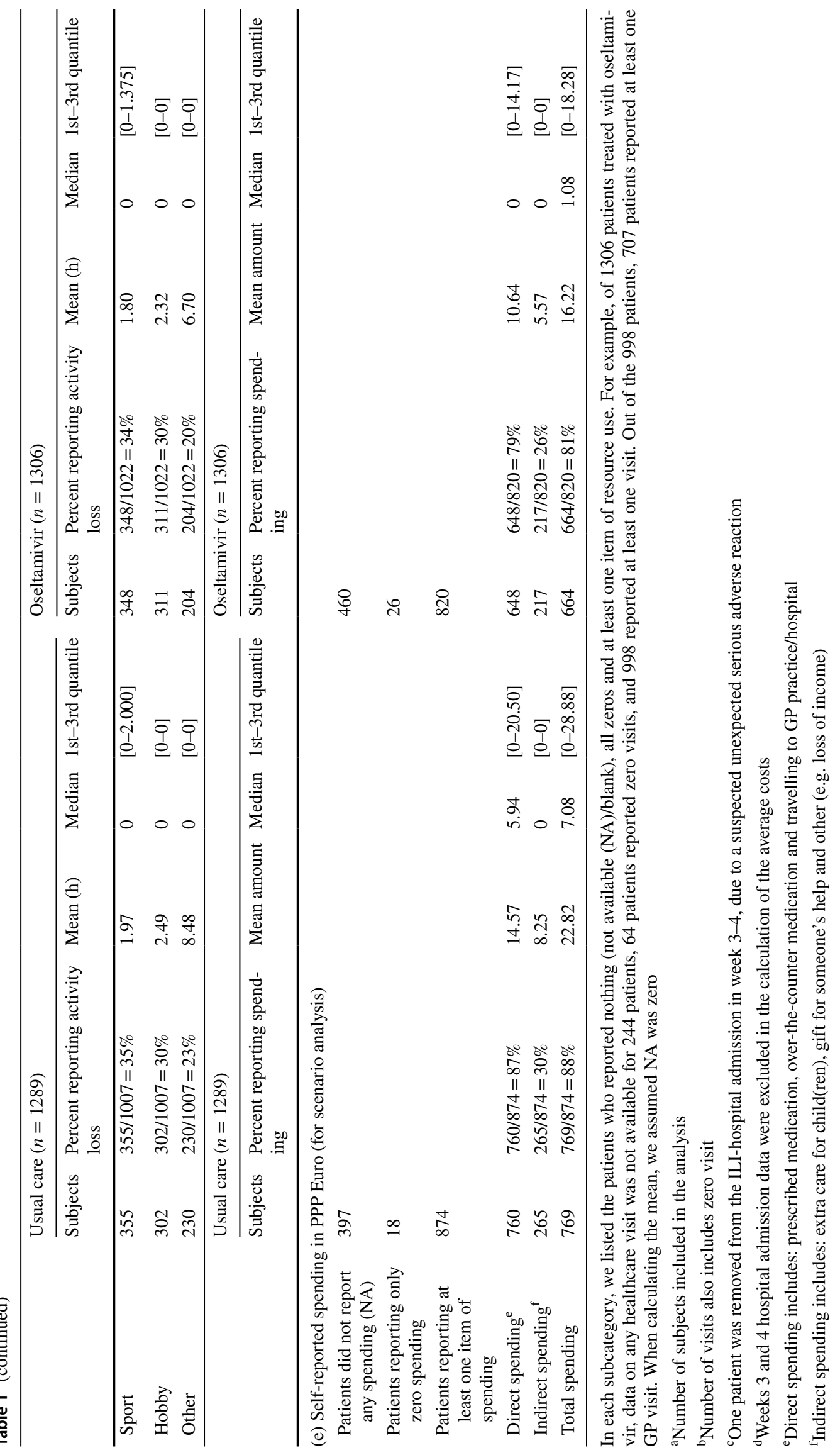


Table 2 Descriptive analysis: average direct, indirect and total costs per influenza-like illness (ILI) patient [median] (1st-3rd quartile) from four perspectives (in €PPP 2018 value) (sample size: usual care $N=1289$ vs. oseltamivir $N=1306$ )

\begin{tabular}{|c|c|c|c|c|c|c|c|c|}
\hline \multirow{2}{*}{$\begin{array}{l}\text { Perspectives } \\
\text { Arms }\end{array}$} & \multicolumn{2}{|l|}{ Patients } & \multicolumn{2}{|c|}{ Healthcare providers } & \multicolumn{2}{|c|}{ Healthcare payers } & \multicolumn{2}{|l|}{ Societal } \\
\hline & Usual care & Oseltamivir & Usual care & Oseltamivir & Usual care & Oseltamivir & Usual care & Oseltamivir \\
\hline \multicolumn{9}{|l|}{ Base case } \\
\hline $\begin{array}{l}\text { Healthcare } \\
\text { visits }\end{array}$ & $\begin{array}{l}1.56[0] \\
(0-0)\end{array}$ & $\begin{array}{l}1.48[0] \\
(0-0)\end{array}$ & $\begin{array}{l}23.00 \text { [7.03] } \\
(0-24.22)\end{array}$ & $\begin{array}{l}22.05 \text { [7.03] } \\
(0-22.65)\end{array}$ & $\begin{array}{l}24.51[7.9] \\
(0-30.9)\end{array}$ & $\begin{array}{l}23.44[7.9] \\
(0-28.8)\end{array}$ & & \\
\hline Medication & $\begin{array}{l}18.32[14.49] \\
(5.52-27.25)\end{array}$ & $\begin{array}{l}15.62[12.53] \\
(4.95-22.62)\end{array}$ & $\begin{array}{l}1.36[0] \\
(0-2.04)\end{array}$ & $\begin{array}{l}1.22[0] \\
(0-2.04)\end{array}$ & $\begin{array}{l}19.63[15.7] \\
(7.35-28.41)\end{array}$ & $\begin{array}{l}16.81[13.43] \\
(5.87-23.83)\end{array}$ & & \\
\hline $\begin{array}{l}\text { Hospital day } \\
\text { visits and } \\
\text { admissions }\end{array}$ & $\begin{array}{l}4.14[0] \\
(0-0)\end{array}$ & $\begin{array}{l}0.05[0] \\
(0-0)\end{array}$ & $\begin{array}{l}19.47[0] \\
(0-0)\end{array}$ & $\begin{array}{l}13.96[0] \\
(0-0)\end{array}$ & $\begin{array}{l}24.41[0] \\
(0-0)\end{array}$ & $\begin{array}{l}14.01[0] \\
(0-0)\end{array}$ & & \\
\hline $\begin{array}{l}\text { Average } \\
\text { direct } \operatorname{cost}^{\mathrm{a}}\end{array}$ & $\begin{array}{l}24.01[16.04] \\
(6.44-29.8)\end{array}$ & $\begin{array}{l}17.15[13.43] \\
(5.17-24.45)\end{array}$ & $\begin{array}{l}43.83[8.2] \\
(1.17-30.48)\end{array}$ & $\begin{array}{l}37.24[7.9] \\
(0-28.77)\end{array}$ & $\begin{array}{l}68.55[34.28] \\
(14.45-55.85)\end{array}$ & $\begin{array}{l}54.26 \text { [29.54] } \\
(12.9-50.8)\end{array}$ & & \\
\hline $\begin{array}{l}\text { Productivity } \\
\text { loss }\end{array}$ & & & & & & & $\begin{array}{c}382.39 \\
{[162.84]} \\
(0-660.58)\end{array}$ & $\begin{array}{l}369.00[157.58] \\
(0-651.24)\end{array}$ \\
\hline $\begin{array}{l}\text { Average total } \\
\text { cost }\end{array}$ & & & & & & & $\begin{array}{c}450.94 \\
{[245.28]} \\
(34.76-702.34) \\
\end{array}$ & $\begin{array}{l}423.26[216.28] \\
(28.49-681.48)\end{array}$ \\
\hline Perspectives & \multicolumn{2}{|l|}{ Patients } & \multicolumn{2}{|c|}{ Healthcare providers } & \multicolumn{2}{|c|}{ Healthcare payers } & \multicolumn{2}{|l|}{ Partial societal } \\
\hline Arms & Usual care & Oseltamivir & Usual care & Oseltamivir & Usual care & Oseltamivir & Usual care & Oseltamivir \\
\hline \multicolumn{9}{|c|}{ Scenario analysis } \\
\hline $\begin{array}{l}\text { Self-reported } \\
\text { direct cost }\end{array}$ & $\begin{array}{l}14.57[5.94] \\
(0-20.5)\end{array}$ & $\begin{array}{l}10.64[0] \\
(0-14.17)\end{array}$ & $\mathrm{NA}^{\mathrm{b}}$ & $\mathrm{NA}^{\mathrm{b}}$ & $\begin{array}{l}14.57[5.94] \\
(0-20.5)\end{array}$ & $\begin{array}{l}10.64[0] \\
(0-14.17)\end{array}$ & & \\
\hline $\begin{array}{l}\text { Average } \\
\text { direct } \operatorname{cost}^{\mathrm{b}}\end{array}$ & $\begin{array}{l}20.26[7.66] \\
(0-23.38)\end{array}$ & $\begin{array}{l}12.17[4.3] \\
(0-15.93)\end{array}$ & $\begin{array}{l}42.47[7.9] \\
(0-28.7)\end{array}$ & $\begin{array}{l}36.02[7.03] \\
(0-28.7)\end{array}$ & $\begin{array}{l}63.49[26.19] \\
(3.54-50)\end{array}$ & $\begin{array}{l}48.1[21.73] \\
(0-43.5)\end{array}$ & & \\
\hline $\begin{array}{l}\text { Self-reported } \\
\text { indirect cost }\end{array}$ & & & & & & & $\begin{array}{l}8.25[0] \\
(0-0)\end{array}$ & $\begin{array}{l}5.57[0] \\
(0-0)\end{array}$ \\
\hline $\begin{array}{l}\text { Average total } \\
\text { cost }\end{array}$ & & & & & & & $\begin{array}{l}71.74[30.47] \\
(4.3-61.71) \\
\end{array}$ & $\begin{array}{l}53.67[22.71] \\
(0-49.33) \\
\end{array}$ \\
\hline \multicolumn{9}{|c|}{ Cost at recruitment stage (cost not included in base-case or scenario analyses) } \\
\hline $\begin{array}{l}\text { Cost of initial } \\
\text { GP visit }\end{array}$ & 1.75 & & 22.32 & & 23.97 & & & \\
\hline $\begin{array}{l}\text { Cost of } \\
\text { oseltamivir }^{\mathrm{a}}\end{array}$ & NA & 24.23 & NA & 2.29 & NA & 26.51 & & \\
\hline
\end{tabular}

${ }^{a}$ Cost of oseltamivir was not included in our analysis

${ }^{\mathrm{b}}$ The costs to healthcare providers were unknown, because patients did not report the costs from healthcare providers' perspective

${ }^{\mathrm{c}}$ In the trial setting, patients were recruited during their initial GP visit, this cost was the same in both groups

\subsection{Average Direct, Indirect and Total Cost per ILI Patient}

The descriptive analysis of average direct, indirect and total costs per patient are presented in Table 2. For the oseltamivir group, the average direct costs were $€ 17, € 37$ and $€ 54$ from the patient's, healthcare provider's and healthcare payers' perspective, respectively. Adding the average indirect costs of $€ 369$, the total cost per ILI patient treated with oseltamivir was $€ 423$ (median $€ 216$, interquartile range $€ 28-681$ ). The average costs for the usual care group were higher for all four perspectives in the base-case analysis (average direct costs were $€ 24, € 44$ and $€ 69$, total cost $€ 451$, respectively), as well as in the scenario analysis (Table 2).

\subsubsection{Statistical Analysis}

Average direct and total costs per patient treated with oseltamivir are not significantly lower than per patient treated with usual care, from all four perspectives, in both the base-case and the scenario analyses (overlapping onesided CrIs, Fig. 2). 

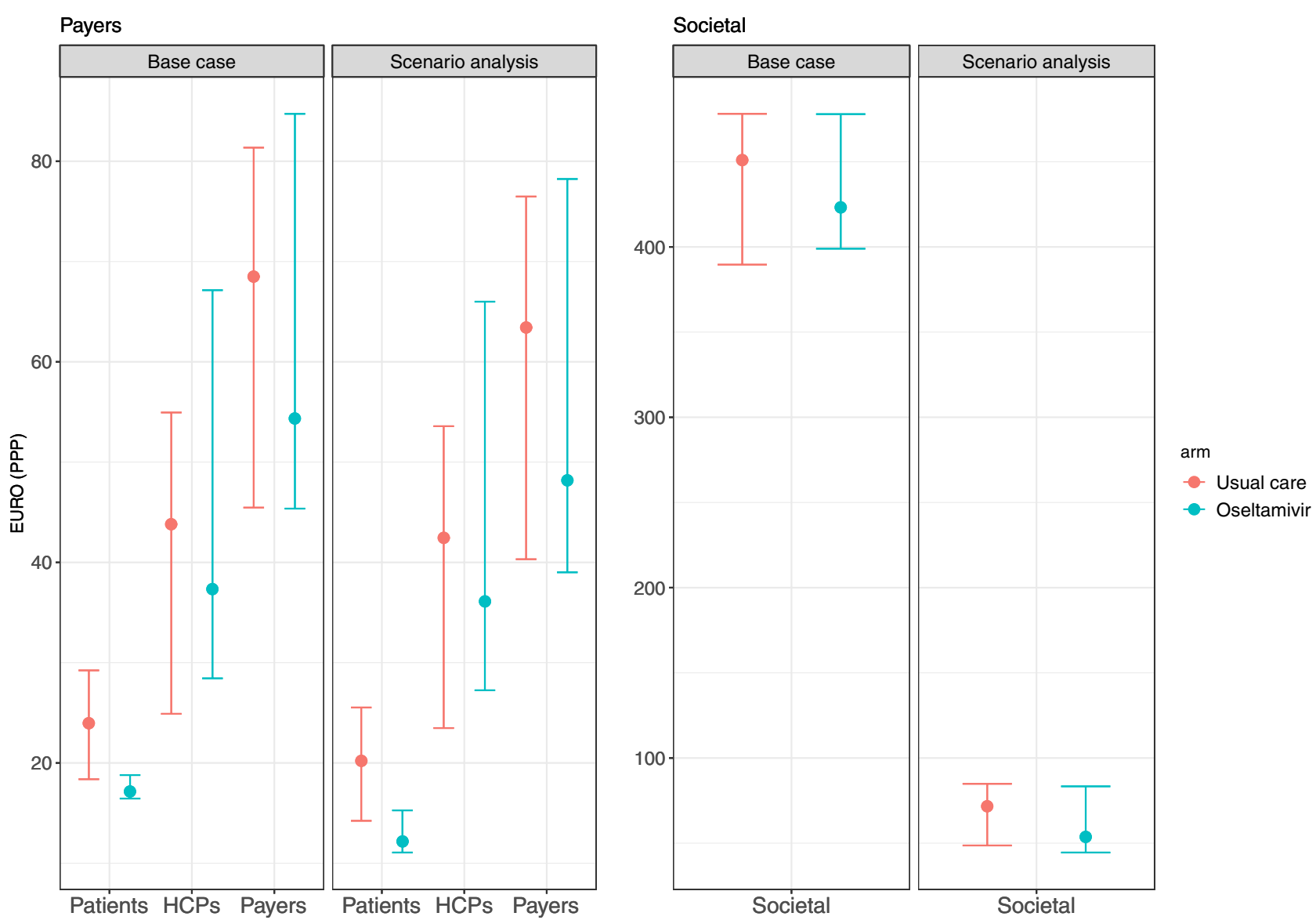

Fig. 2 One-sided credible intervals based on bootstrap (sample size $n=10,000$ ) under different payers' (including patients, healthcare providers (HCPs), healthcare payers) perspectives and a societal perspective. The cost of oseltamivir was not included

\subsubsection{Average Total Cost per ILI Patient by Age Group}

Table 3 presents the average cost per ILI patient for adults/ adolescents and children separately. Average direct and total costs are lower in the oseltamivir compared to the usual care group, for both age groups and from all four perspectives. In general, average costs are lower for children compared to adults/adolescents, especially in the oseltamivir group. In scenario analysis, children had higher average costs from a partial societal perspective (usual care $€ 77$ vs. oseltamivir $€ 58)$ than adults/adolescents (€71 vs. €53).

\subsubsection{Average Total Cost per ILI Patient by Country}

Figure 3 presents the average total cost per country from a societal perspective, base-case analysis. Results for different perspectives and scenario analysis are presented in Supplemental Material, Online Resource 1, Figs. 2-4. Average costs are consistently lower in the oseltamivir group from the four perspectives for five countries (Belgium, Hungary, Poland, Sweden, and the UK), including four of the six countries with the largest sample size ( $>90$ per treatment group).

Two countries with a sample size greater than 90 per treatment group showed opposite findings from the healthcare payers' and societal perspectives. In Lithuania, although the average direct costs are higher in the oseltamivir group from the healthcare payers' perspective (in both base-case and scenario analysis, see Supplemental Material, Online Resource 1, Figs. 3 and 4), the average total costs are lower in the oseltamivir group from a societal perspective (Fig. 3). In Spain, the average total costs are higher in the oseltamivir group from a societal perspective, but the average direct costs are lower (from the healthcare payers' perspective).

Cost of productivity loss accounted for the majority of the total cost in all countries except Sweden and Greece. Sweden has the highest costs of healthcare visits and hospital attendance amongst all countries (e.g. a Swedish GP visit cost is tenfold higher than in Norway and 28 -fold higher than in the Czech Republic). In Greece, the hospital admission costs were proportionately the largest in the oseltamivir group. Notably, the cost of primary healthcare visits in 
Table 3 Average direct and total costs per influenza-like illness (ILI) patient with oseltamivir and usual care amongst two age groups: adults/ adolescents and children (€PPP in 2018 value)

\begin{tabular}{|c|c|c|c|c|c|c|c|}
\hline \multirow[t]{2}{*}{ Perspectives } & \multirow[t]{2}{*}{ Arms } & \multicolumn{3}{|c|}{$\begin{array}{l}\text { Adults/adolescents: over } 13 \text { years of age }(N= \\
\text { 2212) }\end{array}$} & \multicolumn{3}{|c|}{ Children: under 13 years of age $(N=383)$} \\
\hline & & Mean & Median & 1st-3rd quartile & Mean & Median & 1st-3rd quartile \\
\hline \multicolumn{8}{|l|}{ Base case } \\
\hline \multirow[t]{2}{*}{ Patients } & Usual care & 24.91 & 16.05 & $6.44-30.08$ & 18.81 & 15.01 & $6.25-28.39$ \\
\hline & Oseltamivir & 17.23 & 13.71 & $5.31-24.45$ & 16.68 & 13.02 & $4.15-24.29$ \\
\hline \multirow[t]{2}{*}{ Healthcare providers } & Usual care & 43.59 & 8.20 & $1.34-29.49$ & 45.21 & 9.47 & $0-35.05$ \\
\hline & Oseltamivir & 37.75 & 7.90 & $0.65-28.7$ & 34.22 & 8.47 & $0-32.71$ \\
\hline \multirow[t]{2}{*}{ Healthcare payers } & Usual care & 69.34 & 34.31 & $14.86-54.68$ & 64.01 & 32.11 & $12.71-57.12$ \\
\hline & Oseltamivir & 54.84 & 29.85 & $13.39-51.12$ & 50.90 & 28.35 & $10.27-50.39$ \\
\hline \multirow[t]{2}{*}{ Societal } & Usual care & 476.02 & 302.28 & $37.33-726.22$ & 306.77 & 56.65 & $23.30-336.91$ \\
\hline & Oseltamivir & 446.14 & 276.82 & $30.15-707.92$ & 290.48 & 52.08 & $16.06-326.51$ \\
\hline \multicolumn{8}{|l|}{ Scenario analysis } \\
\hline \multirow[t]{2}{*}{ Patients } & Usual care & 20.76 & 7.81 & $0-22.73$ & 17.40 & 6.93 & $0-25.27$ \\
\hline & Oseltamivir & 12.06 & 4.56 & $0-16.53$ & 12.81 & 0 & $0-12.89$ \\
\hline \multirow[t]{2}{*}{ Healthcare providers } & Usual care & 42.16 & 7.90 & $0-28.55$ & 44.25 & 9.47 & $0-34.38$ \\
\hline & Oseltamivir & 36.53 & 7.03 & $0-28.55$ & 33.03 & 7.03 & $0-31.84$ \\
\hline \multirow[t]{2}{*}{ Healthcare payers } & Usual care & 63.81 & 24.25 & $4.3-48.91$ & 61.65 & 32.23 & $0-61.19$ \\
\hline & Oseltamivir & 48.49 & 22.46 & $0-43.5$ & 45.84 & 19.23 & $0-43.51$ \\
\hline \multirow[t]{2}{*}{ Partial societal } & Usual care & 70.88 & 28.70 & $4.31-58.73$ & 76.69 & 40.59 & $3.51-85.88$ \\
\hline & Oseltamivir & 52.95 & 23.00 & $0-47.48$ & 57.87 & 22.5 & $0-56.25$ \\
\hline
\end{tabular}

Greece were the second lowest, but hospitalisations were relatively high (two adults in the oseltamivir group were hospitalised, the cost per patient was €4,207 (PPP)) [20].

\section{Discussion}

This is the first study comparing ILI-associated direct and indirect costs between patients treated with oseltamivir added to usual care and patients treated with usual care only using data collected prospectively from a multi-country, multi-season, randomised trial. When excluding the cost of the oseltamivir itself, we found the average direct and total costs to be lower in the oseltamivir group than in the usual care group, but these differences were not statistically significant. Moreover, when evaluating the associated costs for each country separately, average total costs for the oseltamivir group were lower compared to the usual care group in only seven out of 15 countries from a societal perspective (excluding the oseltamivir cost). However, the per-country sample size was rather small, so larger studies are necessary to demonstrate a potential significant effect of oseltamivir treatment on healthcare use and treatment costs in specific countries.

Our base case estimated higher direct and indirect costs than the scenario analysis in which self-reported spending was included. Our base-case assumption that patients reporting certain medication use incurred the costs of a complete medication package may have led to an overestimation of the medication costs, as patients might have used medication they purchased previously. Moreover, patients may not be directly affected by the losses of paid-work hours (e.g. they received sick pay), which could have resulted in lower indirect costs in our scenario analysis.

If the cost of oseltamivir were included (not shown), we only found statistically significant differences from the patients' perspective: those receiving oseltamivir incurred significantly greater direct costs, because in most countries, oseltamivir would be paid for by the patients. Although nonsignificant, the oseltamivir group incurred greater average direct costs from the healthcare payers' perspective, but the average total costs from the societal perspective were similar between the two groups. In other words, the use of oseltamivir resulted neither in clear excess costs (except for the patient's perspective), nor in net savings. The complete distribution of these costs would need to be evaluated in a subsequent cost-effectiveness analysis.

A Canadian cost-effectiveness model, using data pooled from published trials on healthy adults, demonstrated similar findings: patients receiving oseltamivir reported less antibiotic use and hospitalisations, resulting in lower direct costs when excluding oseltamivir cost (no statistical testing) [21]. A retrospective health insurance claims data analysis in the USA also found that patients receiving oseltamivir incurred 

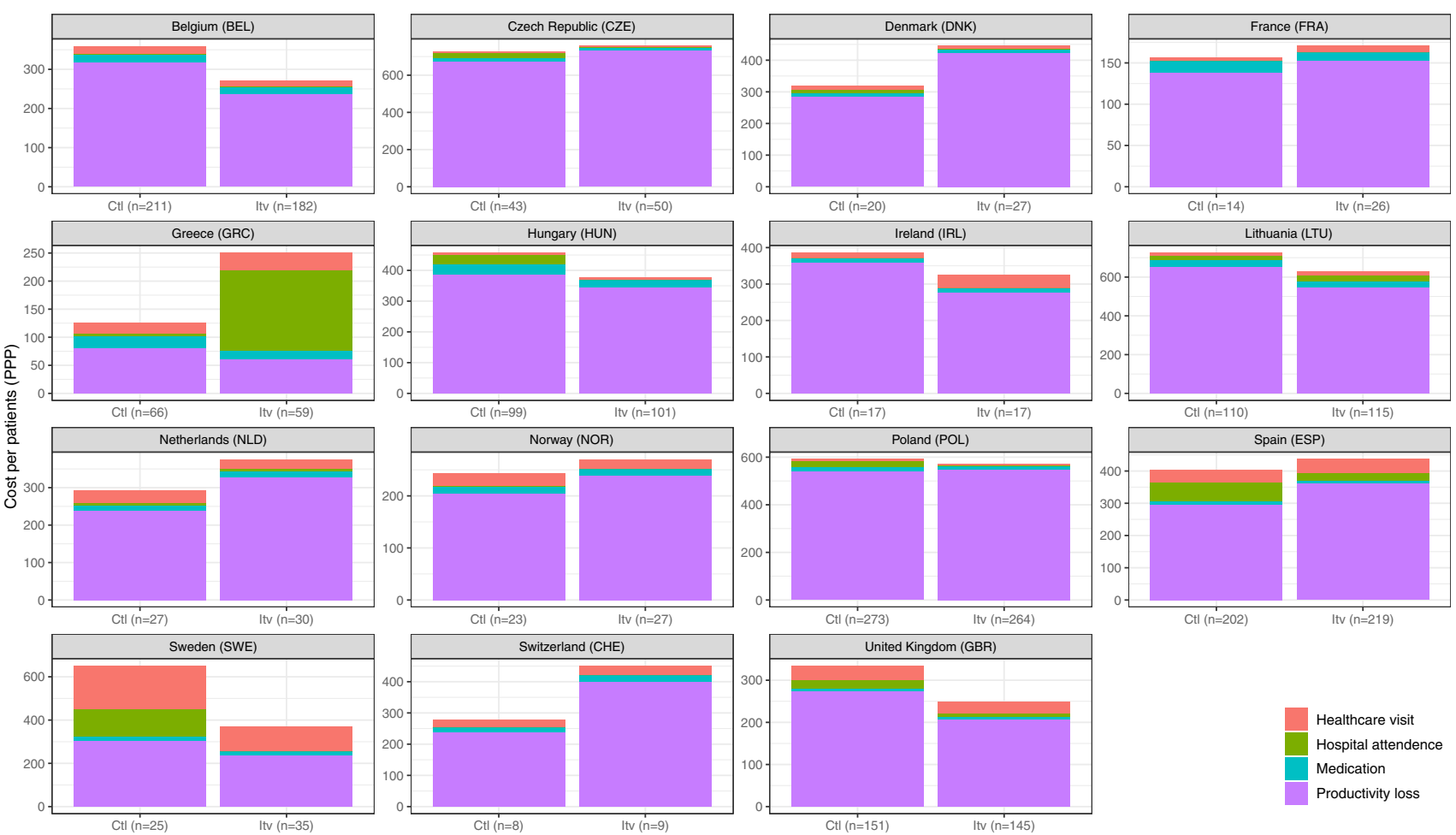

$\mathrm{Ctl}(\mathrm{n}=202)$

$\operatorname{ltv}(n=219)$

Fig. 3 Average cost (€PPP) per country from a societal perspective (pooled adults and children) in the base-case analysis. Ctl usual care group, $l t v$ oseltamivir group. The scale of the $Y$-axis differs between countries

fewer physician and A\&E visits, as well as fewer hospitalisations and shorter LoS if hospitalised, resulting in statistically significant lower direct healthcare costs compared to patients without oseltamivir [22]. Oppong and colleagues estimated on average one to two subsequent GP visits for acute cough/ lower respiratory tract infections (LRTIs) in adults in 13 European countries using diary data over 28 days [23]. This is greater than our estimate $(0.85$ oseltamivir vs. 0.89 usual care, excluding recruitment visit) over 14 days. In contrast to the findings by Oppong and colleagues about healthseeking behaviour after a consultation for a LRTI, we found more out-of-hours, specialist and pharmacy visits after a consultation for ILI. A Belgian ILI burden study reported 1.6 physician visits, which is slightly lower than the mean of 1.85 (oseltamivir) and 1.89 (usual care) GP visits (all countries, including the initial visit). It also estimated 4 days of absence from work or school, which is much higher than the hour losses of paid-work ( 20 vs. $21 \mathrm{~h}$ ) and education ( 3 vs. 4 h) in our analysis; however, we did not include absenteeism before the recruitment date [24].

In Europe, the healthcare systems are vastly different across countries (even regions), which results in difficulties in analysing the direct and indirect costs in a multicountry setting. In some countries, the government provides healthcare through income tax payments (Beveridge model, e.g. the UK), in other countries, people pay a fee to a fund that in turn pays healthcare activities (Bismarck model, e.g. Switzerland). Moreover, both systems are in place in some countries (e.g. France). Consequently, these varying healthcare systems result in considerable differences in health-service delivery, patients' health-seeking behavior, and many other aspects. Firstly, primary healthcare settings can roughly be distinguished into two types: GP-led primary care as gatekeeper to secondary care (e.g. the Netherlands), or a combined system, in which for instance paediatricians also provide primary care for children (e.g. Belgium). Secondly, the payment methods for healthcare providers are also different. Some countries, such as the UK, have a "feeper-service", whereas others like Hungary provide a lump sum payment per registered patient. Consequently, not all countries have official tariffs that provide clear unit costs per healthcare service type. Thirdly, from a patient's perspective, healthcare services were provided free of charge in some countries (e.g. Spain), whereas patients need to pay copayments in other countries (e.g. Switzerland). Furthermore, the methods of estimating unit cost differ according to the country's pharmacoeconomic guideline (if available). For example, fixed costs were excluded in the unit cost estimation in some countries (e.g. in Norway, GPs also received an annual fixed payment per patient on top of payment per visit), but others included such fixed costs (e.g. in Sweden, the unit costs accounted for the annual fixed payment and costs for buildings/premises). One of the strengths of our analysis is that we collected unit costs from four perspectives 
to reflect the cost variations in the real-life setting as much as possible.

Our analysis has several additional strengths. Firstly, detailed country-specific ILI-associated healthcare resource use and costs were reported (in Supplemental Material, Online Resource 1). These unit costs can be used in future studies for respiratory illness as well as for non-disease-specific studies (e.g. cost per primary care or secondary care visit). Secondly, patient-level resource use data were used to estimate the differences between the two groups to reflect the real-world setting. Thirdly, we show results using four different perspectives and did extensive scenario and subgroup analysis, showing the sensitivity of our findings to different assumptions and approaches.

Our analysis also has limitations. Only ILI patients seeking primary healthcare within $72 \mathrm{~h}$ of symptoms onset were recruited; patients with long-lasting and/or with very severe ILI symptoms (direct hospitalisation) were not recruited. This might have led to an underestimation of the average costs, and limited external validity of the findings, because the costs might not represent the total population of ILI patients. Next, our data were collected via diaries for 14 days after the recruitment visit and telephone calls on days 14 and 28. In this cost analysis, we focused on the costs of the first 14 days, and costs thereafter were not included, in keeping with the WHO definition [2]. Therefore, one SUSAR that occurred in the oseltamivir arm after 14 days and would lead to long-term direct and indirect costs was not taken into account. Moreover, the relation between the SUSAR and oseltamivir was due to the time frame, while the SUSAR could very well have been caused by the influenza infection itself. Additionally, unit cost data for adults and adolescents were not separated; however, patients under 18 years of age may pay less or have no co-payment in several healthcare systems. Thereby, we might have overestimated the costs from the patients' perspective among patients 13-18 years of age, who accounted for $5 \%$ of participants (oseltamivir: 71 vs. usual-care groups: 70). Furthermore, some countries set a ceiling for patients' out-of-pocket expenditure for prescribed medication and/or primary and secondary healthcare visits to support patients with chronic conditions (e.g. Sweden). We might therefore have overestimated the costs from the patient's perspective, as the excess costs were covered by healthcare providers; we assumed that the full costs were incurred by these patients, as we had no information on which patients had reached their ceiling (if any). Finally, the healthcare resources provided to patients were not derived from national registries or databases, but from patients' and caregivers' self-report diaries, thus recall bias can be a limitation in this study design.

The collection of unit costs was challenging, especially for costs of out-of-hour services and A\&E visits (not available for 10 and six out of 15 countries, respectively).
Conservative assumptions were made that the cost per outof-hour service was the same as a GP visit and the cost per $A \& E$ visit equalled that of a specialist visit. Although these assumptions could underestimate the average direct costs in both groups, only $6 \%$ and $1 \%$ of patients reported such visits; therefore, the impacts on the overall findings will be limited. In view of this, we recommend that in multi-country analyses the unit costs per country are reported by researchers, rather than only the weighted costs.

Despite all 15 countries having a publicly funded healthcare system, many countries have mixed private and public health insurance (e.g. Ireland, France), as well as mixed types of private and public clinics. We collected the unit cost from public healthcare facilities, as the majority of the patients were covered by public health insurance (oseltamivir $91 \%$ vs. usual care $93 \%$ ).

In most economic analyses, ILI-associated medication costs are often ignored due to limited data or are assumed to be negligible. This is the first study that searched and documented the OTC costs per country, via online pharmacies, and thereby quantified the country-specific cost per pack among 15 European countries. Although the unit cost collection approach requires making assumptions, it gives an indication of OTC prices across countries. Our basecase analysis demonstrated that the OTC costs accounted for a large proportion of direct costs, especially from the patient's perspective. Patients in the usual care group bought more OTC medicines, leading to $17 \%$ higher average outof-pocket expenditure $(€ 18.32)$ than the oseltamivir group $(€ 15.62)$. Ignoring the OTC costs might considerably underestimate the incremental costs from the patients' and healthcare payers' perspectives, as well as the total ILI-associated disease burden.

\section{Conclusion}

Our analysis demonstrated that the average direct and total costs of ILI patients treated with oseltamivir were consistently lower than these costs of ILI patients treated with usual care alone from patients', healthcare providers', healthcare payers' and societal perspectives, when excluding the cost of oseltamivir. However, these differences were not statistically significant, and between-country differences were observed. Larger studies are needed to estimate country-specific cost differences.

Supplementary Information The online version contains supplementary material available at https://doi.org/10.1007/s40261-021-01057-y.

Acknowledgements We would like to thank the following $\mathrm{ALIC}^{4} \mathrm{E}$ country data contributors; Belgium: Joris Maesschalck for providing Belgian Pharmacy data (market share and price), and Dr. Annelies Colliers for trial co-ordination and clinical data/study diary collection. 
Spain: Helena Pera for collecting unit cost data, Dr. Ana Moragas and Dr. Carl Llor for reviewing the data in Catalonia. Hungary: Dr. Bernadett Kovács for reviewing unit cost data. Sweden: Lars Levin for providing unit cost data. Norway: Prof. Morten Lindbæk for reviewing the unit cost data. Netherlands: Prof. Theo Verheij for reviewing the unit cost data. Ireland: Eileen Fahey for reviewing the unit cost data. France: Dr. Pia Touboul Lundgren for reviewing the unit cost data, and Chistine Pintaric for trial co-ordination and clinical data/study diary collection. Clinical trial data management team: Susan van Hemert. We also thank all the clinicians and patients for participating in the $\mathrm{ALIC}^{4} \mathrm{E}$ trial.

\section{Declarations}

Funding The $\mathrm{ALIC}^{4} \mathrm{E}$ trial and the contributions of $\mathrm{AWV}, \mathrm{EB}, \mathrm{SC}, \mathrm{NF}$, PS, NJH, PaB, AGS, DG, KK, RMP, RRJ, BS, NT, RA, CCB and PB were funded by the European Commission's Seventh Framework Programme: Platform for European Preparedness Against (Re-)emerging Epidemics (PREPARE) (grant HEALTH-F3-2013-602525). PB, XL, JB and RB acknowledge partial Methusalem funding from the VAXIDEA and ASCID Centres of Excellence in vaccination and infectious diseases at the University of Antwerp. JB and RB were funded by their personal postdoctoral grants from the Research FoundationFlanders (FWO). PS acknowledges that The Healthcare Committee, Region Västra Götaland, Sweden, partially funded the Swedish part of the study.

Conflict of interest NF reports grants from Synairgen outside the submitted work. CCB reports grants from National Institute for Health Research Health and grants from National Institute for Health Research Health Protection Research Unit on Health Care Associated Infections and Antimicrobial Resistance during the conduct of the study; personal fees from Roche Molecular Systems, grants from Roche Molecular Diagnostics, personal fees from Pfizer, personal fees from Roche diagnostics, personal fees from Janssen Pharmaceuticals, outside the submitted work. DG reports being employed since 1 June 2020 by Roche Pharma (Schweiz) AG, Basel, Switzerland. Data collections for this articlet were completed before his current employment. The current employment did not influence this article. PB reports grants from European commission IMI, outside the submitted work. XL, JB, AWV, EB, RB, SC, PS, NJH, PaB, AGS, KK, RMP, RRJ, BS, NT and RA report no conflicts of interest.

Ethics approval The study was approved by the National Research Ethics Service Committee South Central-Oxford B. Clinical trial authority approval was obtained from the UK Medicines and Healthcare products Regulatory Agency. Per participating country appropriate institutional, national research ethics committee and regulatory approvals were obtained. The $\mathrm{ALIC}^{4} \mathrm{E}$ trial was performed in accordance with the ethical standards as laid down in the 1964 Declaration of Helsinki and its later amendments or comparable ethical standards.

Consent to participate All trial participants provided written informed consent.

\section{Consent for publication Not applicable.}

Availability of data and material Almost all the data analysed and generated during this study are included in this published article and its supplementary information files. Formal requests for additional data can be made to the corresponding author (XL) or the senior authors (AWV, PB) using a bespoke data request form delineating research aims, methods and the variables.

Code availability Not applicable.
Author contributions All authors made substantial contributions to the design of the work and interpretation of data. CCB was the chief investigator of this trial. CCB, AWV and EB led the development of the clinical trial, and managed and coordinated the trial. $\mathrm{PB}, \mathrm{XL}$ and JB designed the unit cost collection templates. PS, NH, SC, NF, PaB, AGS, DG, KK, RMP, RRJ, BS, NT, RA and AWV were responsible for their network's participation in the trial, and collected or reviewed the country-specific unit costs. XL performed the literature searches, data collection and data analyses. XL and JB wrote the initial draft. PB, RB, $\mathrm{CCB}$ and $\mathrm{AWV}$ contributed to the analyses and substantively revised the manuscript. All authors critically reviewed and commented on previous versions of the manuscript. All authors have read and approved the submitted version and agreed to be accountable for all aspects of the work in ensuring that questions related to the accuracy or integrity of any part of the work are appropriately investigated and resolved.

Open Access This article is licensed under a Creative Commons Attribution-NonCommercial 4.0 International License, which permits any non-commercial use, sharing, adaptation, distribution and reproduction in any medium or format, as long as you give appropriate credit to the original author(s) and the source, provide a link to the Creative Commons licence, and indicate if changes were made. The images or other third party material in this article are included in the article's Creative Commons licence, unless indicated otherwise in a credit line to the material. If material is not included in the article's Creative Commons licence and your intended use is not permitted by statutory regulation or exceeds the permitted use, you will need to obtain permission directly from the copyright holder. To view a copy of this licence, visit http://creativecommons.org/licenses/by-nc/4.0/.

\section{References}

1. Collaborators GBDI. Mortality, morbidity, and hospitalisations due to influenza lower respiratory tract infections, 2017: an analysis for the Global Burden of Disease Study 2017. Lancet Respir Med. 2019;7(1):69-89.

2. WHO surveillance case definitions for ILI and SARI. https://www. who.int/influenza/surveillance_monitoring/ili_sari_surveillance_ case_definition/en/.

3. Antonova EN, Rycroft CE, Ambrose CS, Heikkinen T, Principi $\mathrm{N}$. Burden of paediatric influenza in Western Europe: a systematic review. BMC Public Health. 2012;12:968.

4. Federici C, Cavazza M, Costa F, Jommi C. Health care costs of influenza-related episodes in high income countries: a systematic review. PLoS ONE. 2018;13(9):e0202787.

5. National Institute for Health and Care Excellence: amantadine, oseltamivir and zanamivir for the treatment of influenza. 25/02/2009 edn; 2009. https://www.nice.org.uk/guidance/ta168.

6. Public Health England: PHE guidance on use of antiviral agents for the treatment and prophylaxis of seasonal influenza. Public Health England; 2019. https://assets.publishing.service.gov. uk/government/uploads/system/uploads/attachment_data/file/ 833572/PHE_guidance_antivirals_influenza_201920.pdf

7. Influenza antiviral medications: summary for clinicians. https:// www.cdc.gov/flu/professionals/antivirals/summary-clinicians. htm.

8. Adriaenssens N, Coenen S, Kroes AC, Versporten A, Vankerckhoven V, Muller A, Blix HS, Goossens H, Group EP. European Surveillance of Antimicrobial Consumption (ESAC): systemic antiviral use in Europe. J Antimicrob Chemother. 2011;66(8):1897-905.

9. Jefferson T, Jones MA, Doshi P, Del Mar CB, Hama R, Thompson MJ, Spencer EA, Onakpoya I, Mahtani KR, Nunan D, et al. 
Neuraminidase inhibitors for preventing and treating influenza in healthy adults and children. Cochrane Database Syst Rev. 2014(4):CD008965.

10. Dobson J, Whitley RJ, Pocock S, Monto AS. Oseltamivir treatment for influenza in adults: a meta-analysis of randomised controlled trials. Lancet. 2015;385(9979):1729-37.

11. Wang K, Shun-Shin M, Gill P, Perera R, Harnden A. Neuraminidase inhibitors for preventing and treating influenza in children (published trials only). Cochrane Database Syst Rev. 2012(4):CD002744.

12. Shun-Shin M, Thompson M, Heneghan C, Perera R, Harnden A, Mant D. Neuraminidase inhibitors for treatment and prophylaxis of influenza in children: systematic review and meta-analysis of randomised controlled trials. BMJ. 2009;339:b3172.

13. Butler CC, van der Velden AW, Bongard E, Saville BR, Holmes J, Coenen S, Cook J, Francis NA, Lewis RJ, Godycki-Cwirko M, et al. Oseltamivir plus usual care versus usual care for influenzalike illness in primary care: an open-label, pragmatic, randomised controlled trial. Lancet. 2020;395(10217):42-52.

14. Bongard E, van der Velden AW, Cook J, Saville B, Beutels P, Munck Aabenhus R, Brugman C, Chlabicz S, Coenen S, Colliers A, et al. Antivirals for influenza-Like Illness? A randomised Controlled trial of Clinical and Cost effectiveness in primary CarE (ALIC(4) E): the ALIC(4) E protocol. BMJ Open. 2018;8(7):e021032.

15. European Medicines Agency: Summary of product characteristics of Tamiflu (Oseltamivir). Official website European Medicines Agency. https://www.ema.europa.eu/en/medicines/human/EPAR/ tamiflu.

16. Eurostat: Purchasing power parities (PPPs), price level indices and real expenditures for ESA 2010 aggregates[prc_ppp_ind]. https:// appsso.eurostat.ec.europa.eu/nui/show.do?dataset=prc_ppp_ind \& lang=en.
17. World Bank: Consumer price index. https://data.worldbank.org/ indicator/FP.CPI.TOTL.

18. Desgagne A, Castilloux AM, Angers JF, LeLorier J. The use of the bootstrap statistical method for the pharmacoeconomic cost analysis of skewed data. Pharmacoeconomics. 1998;13(5 Pt 1):487-97.

19. Hesterberg T, Monaghan S, Moore D, Clipson A, Epstein R. Bootstrap methods and permutation tests. W. H. Freeman and Company; 2003.

20. Strutton DR, Farkouh RA, Earnshaw SR, Hwang S, Theidel U, Kontodimas S, Klok R, Papanicolaou S. Cost-effectiveness of 13-valent pneumococcal conjugate vaccine: Germany, Greece, and The Netherlands. J Infect. 2012;64(1):54-67.

21. O'Brien BJ, Goeree R, Blackhouse G, Smieja M, Loeb M. Oseltamivir for treatment of influenza in healthy adults: pooled trial evidence and cost-effectiveness model for Canada. Value Health. 2003;6(2):116-25.

22. Gums JG, Pelletier EM, Blumentals WA. Oseltamivir and influenza-related complications, hospitalization and healthcare expenditure in healthy adults and children. Expert Opin Pharmacother. 2008;9(2):151-61.

23. Oppong R, Coast J, Hood K, Nuttall J, Smith RD, Butler CC, Team G-S. Resource use and costs of treating acute cough/lower respiratory tract infections in 13 European countries: results and challenges. Eur J Health Econ. 2011;12(4):319-29.

24. Bilcke J, Coenen S, Beutels P. Influenza-like-illness and clinically diagnosed flu: disease burden, costs and quality of life for patients seeking ambulatory care or no professional care at all. PLoS ONE. 2014;9(7):e102634.

\section{Authors and Affiliations}

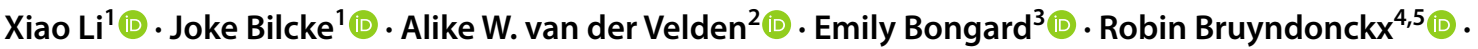 Pär-Daniel Sundvall ${ }^{6,18}$ (D) Nicolay J. Harbin ${ }^{7}$ (I) . Samuel Coenen ${ }^{5}$. Nick Francis ${ }^{8}$ (D) Pascale Bruno $^{9}$. Ana Garcia-Sangenis ${ }^{10}$ (D) Dominik Glinz ${ }^{11}$ - Katarzyna Kosiek ${ }^{12} \cdot$ Réka Mikó-Pauer $^{13}$. Ruta Radzeviciene Jurgute ${ }^{14} \cdot$ Bohumil Seifert $^{15}$ (i) . Nikolaos Tsakountakis ${ }^{16} \cdot$ Rune Aabenhus $^{17}$ (i) . Christopher C. Butler ${ }^{3} \cdot$ Philippe Beutels $^{1}$ (D)}

Joke Bilcke

joke.bilcke@uantwerpen.be

Alike W. van der Velden

A.W.vanderVelden@umcutrecht.nl

Emily Bongard

emily.bongard@phc.ox.ac.uk

Robin Bruyndonckx

robin.bruyndonckx@uhasselt.be

Pär-Daniel Sundvall

par-daniel.sundvall@vgregion.se

Nicolay J. Harbin

n.j.harbin@medisin.uio.no

Samuel Coenen

samuel.coenen@uantwerpen.be

Nick Francis

Nick.Francis@soton.ac.uk
Pascale Bruno

bruno.p@chu-nice.fr

Ana Garcia-Sangenis

agarcia@idiapjgol.org

Dominik Glinz

dominik.glinz@usb.ch

Katarzyna Kosiek

kasia@uni.lodz.pl

Réka Mikó-Pauer

eka.pauer@drc.hu

Ruta Radzeviciene Jurgute

seimosgyd@gmail.com

Bohumil Seifert

bohumil.seifert@1f1.cuni.cz

Nikolaos Tsakountakis

drtsakou@yahoo.gr 
Rune Aabenhus

runa@sund.ku.dk

Christopher C. Butler

christopher.butler@phc.ox.ac.uk

Philippe Beutels

philippe.beutels@uantwerpen.be

1 Centre for Health Economics Research and Modelling Infectious Diseases (CHERMID), Vaccine and Infectious Disease Institute, University of Antwerp, Universiteitsplein 1, 2610 Wilrijk, Belgium

2 Julius Center for Health Sciences and Primary Care, University Medical Center, Utrecht, The Netherlands

3 The Nuffield Department of Primary Care Health Sciences, University of Oxford, Oxford, UK

4 Interuniversity Institute for Biostatistics and statistical Bioinformatics (I-BIOSTAT), Data Science Institute (DSI), Hasselt University, Hasselt, Belgium

5 Department of Family Medicine and Population Health (FAMPOP), Vaccine and Infectious Disease Institute (VAXINFECTIO), Laboratory of Medical Microbiology, University of Antwerp, Antwerp, Belgium

6 Primary Health Care, School of Public Health and Community Medicine, Institute of Medicine, Sahlgrenska Academy, University of Gothenburg, Gothenburg, Sweden

7 Antibiotic Center for Primary Care, Department of General Practice, Institute of Health and Society, University of Oslo, Oslo, Norway
8 School of Primary Care, Population Sciences and Medical Education, Faculty of Medicine, University of Southampton, Southampton, UK

9 Département de Santé Publique, Centre Hospitalier Universitaire de Nice, Université Côte d'Azur, Nice, France

10 Drug Study Unit, Institut Universitari d'Investigació en Atenció Primària Jordi Gol (IDIAP Jordi Gol), Barcelona, Spain

11 Department of Clinical Research, Basel Institute for Clinical Epidemiology and Biostatistics, University Hospital Basel, Basel, Switzerland

12 Family Doctors' Clinic, Lodz, Poland

13 DRC Drug Research Center LLC, Balatonfüred, Hungary

14 FDC Mano šeimos gydytojas, Klaipeda, Lithuania

15 First Faculty of Medicine, Institute of General Practice, Charles University in Prague, Prague, Czech Republic

16 Malia Surgery, Kastelli HC, Heraklio, Greece

17 Section and Research Unit of General Practice, Department of Public Health, University of Copenhagen, Copenhagen, Denmark

18 Research and Development Primary Health Care Region Västra Götaland, Research and Development Centre Södra Älvsborg, Borås, Sweden 